\title{
On Cyclic Cubic Fields
}

\author{
By Veikko Ennola and Reino Turunen
}

\begin{abstract}
A table of class numbers and units in cyclic cubic fields with conductor $<4000$ has been given by Marie-Nicole Gras [6]. The authors have constructed an extended table for conductor $<16000$. The article comprises lists of fields with totally positive fundamental units and fields in which the class group has a Sylow $p$-subgroup which is not elementary abelian. We also give statistics about the distribution of class numbers.
\end{abstract}

Let $K$ be a cyclic cubic field with conductor $f$ and denote by $\mathcal{O}$ the ring of integers of $K$. If $\alpha \in K$, its conjugates are denoted by $\alpha, \alpha^{\prime}, \alpha^{\prime \prime}$ and the trace and norm by $\operatorname{Tr}(\alpha)=\alpha+\alpha^{\prime}+\alpha^{\prime \prime}$ and $\mathrm{N}(\alpha)=\alpha \alpha^{\prime} \alpha^{\prime \prime}$. We can write

$$
f=\left(a^{2}+3 b^{2}\right) / 4
$$

where $a$ and $b$ satisfy the conditions

$$
\begin{array}{lll}
a \equiv 2 \bmod 3, & b \equiv 0 \bmod 3, \quad b>0 & \text { for } 3+f, \\
a \equiv 6 \bmod 9, & b \equiv 3 \operatorname{or} 6 \bmod 9, \quad b>0 & \text { for } 3 \mid f,
\end{array}
$$

introduced by Hasse [8, p. 12]. Let $\theta, \theta^{\prime}, \theta^{\prime \prime}$ denote the Gaussian periods for a generating cubic character of $K$ taken with a suitable sign. Then $K=\mathbf{Q}(\theta)$ and (see, e.g., $[9$, pp. 7-9])

$$
\operatorname{Irr}(\theta, \mathbf{Q})= \begin{cases}x^{3}+x^{2}+((1-f) / 3) x+(f(a-3)+1) / 27 & \text { for } 3+f \\ x^{3}-(f / 3) x-f a / 27 & \text { for } 3 \text { । } f\end{cases}
$$

A unit $\tau$ of the ring $\mathcal{O}$ is called a fundamental unit iff $-1, \tau$ and $\tau^{\prime}$ generate the group of units of $\mathcal{O}$. We shall further stipulate that

$$
\mathrm{N}(\tau)=1 \text { and two of the conjugates of } \tau \text { have absolute value }>1 \text {. }
$$

It is easy to see that any fundamental unit satisfying (3) must be a conjugate of $\tau$ so that their minimal polynomial

$$
\operatorname{Irr}(\tau, \mathbf{Q})=x^{3}-\operatorname{Tr}(\tau) x^{2}+\operatorname{Tr}\left(\tau^{-1}\right) x-1
$$

is uniquely determined.

We note that (3) implies $\left|\operatorname{Tr}\left(\tau^{-1}\right)\right|>|\operatorname{Tr}(\tau)|$. This can be easily seen as follows. Put $s=\operatorname{Tr}(\tau), q=\operatorname{Tr}\left(\tau^{-1}\right)$ and let $\delta$ denote the sign of that conjugate of $\tau$ which has absolute value $<1$. Then, $\delta(s-q)<0$ and $\delta(s+q+2)>0$, so that $|s| \geqslant|q|$ implies $s=-q$ or $s=-q-1$. Under these conditions the discriminant of (4) is a square only for $s=-4, q=3$, but, in this case, $\tau$ would not be a fundamental unit.

Received April 19, 1983; revised March 26, 1984.

1980 Mathematics Subject Classification. Primary 12-04, 12A30, 12A35, 12A45, 12A50. 
Tables containing the coefficients of (4) and the class number $h$ of $K$ have been given by M. N. Gras [6] (i) systematically for $f<4000$, (ii) in the cases $h \equiv 0 \bmod 9$ and $h \equiv 0 \bmod 4$ for $4000<f<10000$, and (iii) for certain particular families of fields with $4000<f<20000$. The choice of the parameters $a, b$ in [6] is different from ours, owing to the fact that we have stuck to Hasse's old normalization (2). Thus, our numbers $a, b$ are the same as $-a, 3 b$ in $\mathrm{M}$. N. Gras's notation. In the tables of M. N. Gras there are a number of gaps which have been filled independently by Godwin [2], whose method is entirely different from ours, and by ourselves. The two results agree exactly.

We have constructed an extended table of the 1906 fields with $4000<f<16000$. (The total number of cyclic cubic fields with $f<16000$ is $630+1906=2536$.) The table is deposited in the Mathematics of Computation's UMT-depository. It consists of three parts, the $k$ th part containing the fields with $4000 k<f<4000(k+1)$. For each field $K$ the following data are given:

- running number $r$

- conductor $f$

- parameters $a, b$

- class number $h$

- coefficients $\operatorname{Tr}(\tau), \operatorname{Tr}\left(\tau^{-1}\right)$ of the polynomial (4).

The number $\operatorname{Tr}\left(\tau^{-1}\right)$ is written underneath $\operatorname{Tr}(\tau)$ in case there is not enough space for both of them on the same line. The fields are arranged lexicographically according to increasing values of $f$ and $b$.

The method employed in the computation is essentially the same adaptation of the Voronoi algorithm that we used in [1] to construct a table of totally real cubic fields. In the cyclic case, the unit lattice in the logarithmic space is hexagonal and, therefore, the reduced units determined by our algorithm are conjugates and thus both fundamental units. In fact, one of our reliability tests is based on calculating both units separately and on checking the equality of the minimal polynomials. A disadvantage of the Voronoi algorithm is the fact that the units produced by it

TABLE 1

\begin{tabular}{rrrrrrrrrrrrr}
\hline \multicolumn{1}{c}{$f$} & \multicolumn{1}{c}{$a$} & \multicolumn{1}{c}{$b$} & \multicolumn{1}{c}{$h$} & \multicolumn{1}{c}{$f$} & \multicolumn{1}{c}{$a$} & \multicolumn{1}{c}{$h$} & \multicolumn{1}{c}{$f$} & \multicolumn{1}{c}{$a$} & \multicolumn{1}{c}{$b$} & $h$ \\
\hline 703 & -25 & 27 & 12 & 711 & -12 & 30 & 12 & 1009 & -43 & 27 & 4 \\
1699 & -64 & 30 & 4 & 3193 & -55 & 57 & 12 & 4291 & -64 & 66 & 12 \\
4357 & 119 & 33 & 4 & 4561 & -37 & 75 & 4 & 4639 & 41 & 75 & 4 \\
5257 & -115 & 51 & 12 & 6037 & -154 & 12 & 4 & 6289 & -91 & 75 & 12 \\
6381 & -93 & 75 & 12 & 6553 & 155 & 27 & 4 & 7027 & 161 & 27 & 4 \\
7639 & -172 & 18 & 4 & 8029 & -178 & 12 & 36 & 8191 & 92 & 90 & 4 \\
8557 & 179 & 27 & 12 & 8659 & -169 & 45 & 12 & 8797 & 14 & 108 & 84 \\
9109 & 38 & 108 & 4 & 9217 & 146 & 72 & 12 & 9283 & -13 & 111 & 4 \\
9667 & 191 & 27 & 12 & 9667 & -160 & 66 & 12 & 10147 & -121 & 93 & 12 \\
10399 & 23 & 117 & 4 & 10771 & -64 & 114 & 4 & 11241 & -201 & 39 & 12 \\
11257 & 125 & 99 & 4 & 12127 & 185 & 69 & 12 & 12757 & 221 & 27 & 4 \\
13297 & 194 & 72 & 4 & 13459 & 113 & 117 & 12 & 13531 & -193 & 75 & 12 \\
13921 & 197 & 75 & 4 & 14209 & -217 & 57 & 12 & 14287 & -4 & 138 & 36 \\
14449 & 239 & 15 & 4 & 14521 & 122 & 120 & 36 & 14689 & -133 & 117 & 12 \\
14917 & 86 & 132 & 12 & 15139 & 209 & 75 & 4 & 15237 & -246 & 12 & 12 \\
\hline
\end{tabular}


TABLE 2

\begin{tabular}{|c|c|c|c|c|c|c|}
\hline$f$ & $\bar{a}$ & $b$ & $h$ & Generators of $G$ & Relations & Invariants of $G$ \\
\hline 1777 & 14 & 48 & 16 & $\mathrm{Cl} \mathfrak{p}_{2}, \mathrm{Cl}_{2}^{\prime}$ & $\mathfrak{p}_{2}^{4} \sim \mathfrak{p}_{2}^{\prime 4} \sim 1$ & 4,4 \\
\hline 2817 & -66 & 48 & 48 & $\mathrm{Cl}_{2}, \mathrm{Cl} \mathfrak{p}_{2}^{\prime}$ & $\mathfrak{p}_{2}^{12} \sim 1, \mathfrak{p}_{2}^{\prime 4} \sim \mathfrak{p}_{2}^{4}$ & $3,4,4$ \\
\hline 4297 & 131 & 3 & 16 & $\mathrm{Cl} p_{13}, \mathrm{Cl} \mathfrak{p}_{13}^{\prime}$ & $\mathfrak{p}_{13}^{4} \sim \mathfrak{p}_{13}^{4} \sim 1$ & 4,4 \\
\hline 4711 & 113 & 45 & 27 & $\mathrm{Cl}_{3}, \mathrm{Cl} p_{3}^{\prime}$ & $\mathfrak{p}_{3}^{9} \sim 1, \mathfrak{p}_{3}^{\prime 3} \sim \mathfrak{p}_{3}^{3}$ & 3,9 \\
\hline 4711 & -76 & 66 & 27 & $\mathrm{Cl} \mathfrak{p}_{2}, \mathrm{Cl}_{\mathfrak{p}_{2}^{\prime}}$ & $\mathfrak{p}_{2}^{9} \sim 1, \mathfrak{p}_{2}^{\prime 3} \sim \mathfrak{p}_{2}^{3}$ & 3,9 \\
\hline 5383 & 92 & 66 & 27 & $\mathrm{Cl}_{2}, \mathrm{Cl}_{2}^{\prime}$ & $\mathfrak{p}_{2}^{9} \sim 1, \mathfrak{p}_{2}^{\prime 3} \sim \mathfrak{p}_{2}^{3}$ & 3,9 \\
\hline 5383 & -43 & 81 & 27 & $\mathrm{Cl} \mathrm{p}_{3}, \mathrm{Cl} \mathfrak{p}_{3}^{\prime}$ & $\mathfrak{p}_{3}^{9} \sim 1, \mathfrak{p}^{\prime 3}{ }_{3}^{\frac{3}{9}} \sim \mathfrak{p}_{3}^{\overline{3}}$ & 3,9 \\
\hline 5409 & -147 & 3 & 48 & $\mathrm{Cl} p_{11}, \mathrm{Cl} p_{11}^{\prime}$ & $\mathfrak{p}_{11}^{12} \sim 1, \mathfrak{p}_{11}^{\prime 4} \sim \mathfrak{p}_{11}^{4}$ & $3,4,4$ \\
\hline 6139 & -16 & 90 & 48 & $\mathrm{Cl} \mathfrak{p}_{2}, \mathrm{Cl} \mathfrak{p}_{2}^{\prime}$ & $\mathfrak{p}_{2}^{12} \sim 1, \mathfrak{p}_{2}^{\prime 4} \sim \mathfrak{p}_{2}^{4}$ & 3.4 .4 \\
\hline 6247 & -151 & 27 & 16 & $\mathrm{Cl} \mathfrak{p}_{11}, \mathrm{Cl} \mathfrak{p}_{11}^{\prime}$ & $\mathfrak{p}_{11}^{4} \sim \mathfrak{p}_{11}^{4} \sim 1$ & 4,4 \\
\hline 7351 & -1 & 99 & 49 & $\mathrm{Cl} \mathfrak{p}_{3}$ & $\mathfrak{p}_{3}^{49} \sim 1$ & 49 \\
\hline 7657 & 170 & 24 & 81 & $\mathrm{Cl} \mathfrak{p}_{2}, \mathrm{Cl} \mathfrak{p}_{2}^{\prime}, \mathrm{Cl} \mathfrak{p}_{5}$ & $\mathfrak{p}_{2}^{9} \sim 1, \mathfrak{p}_{2}^{\prime 3} \sim \mathfrak{p}_{2}^{3}, \mathfrak{p}_{5}^{3} \sim \mathfrak{p}_{2}^{6}$ & $3,3,9$ \\
\hline 8563 & 185 & 3 & 49 & $\mathrm{Clp}_{5}$ & $\mathfrak{p}_{5}^{49} \sim 1$ & 49 \\
\hline 9247 & -76 & 102 & 48 & $\mathrm{Cl} \mathrm{p}_{2}, \mathrm{Cl} p_{2}^{\prime}$ & $\mathfrak{p}_{2}^{12} \sim 1, \mathfrak{p}_{2}^{4} \sim \mathfrak{p}_{2}^{4}$ & $3,4,4$ \\
\hline 10513 & -205 & 3 & 64 & $\mathrm{Cl} p_{11}, \mathrm{Cl} p_{11}^{\prime}$ & $\mathfrak{p}_{11}^{8} \sim \mathfrak{p}_{11}^{82} \sim 1$ & 8,8 \\
\hline 11167 & -181 & 63 & 27 & $\mathrm{Cl}_{3}, \mathrm{Cl} \mathfrak{p}_{3}^{\prime}$ & $\mathfrak{p}_{3}^{9} \sim 1, \mathfrak{p}_{3}^{\prime 3} \sim \mathfrak{p}_{3}^{3}$ & 3,9 \\
\hline 11167 & 116 & 102 & 27 & $\mathrm{Cl} \mathfrak{p}_{2}, \mathrm{Cl}_{\mathfrak{p}_{2}^{\prime}}$ & $\mathfrak{p}_{2}^{9} \sim 1, \mathfrak{p}_{2}^{\prime 3} \sim \mathfrak{p}_{2}^{3}$ & 3,9 \\
\hline 12403 & 173 & 81 & 27 & $\mathrm{Cl} \mathrm{p}_{3}, \mathrm{Cl}_{\mathfrak{p}_{3}^{\prime}}$ & $\mathfrak{p}_{3}^{9} \sim 1, \mathfrak{p}_{3}^{\prime 3} \sim \mathfrak{p}_{3}^{3}$ & 3,9 \\
\hline 12403 & 65 & 123 & 27 & $\mathrm{Cl} p_{5}, \mathrm{Cl}_{5}^{\prime}$ & $\mathfrak{p}_{5}^{9} \sim 1, \mathfrak{p}_{5}^{\prime 3} \sim \mathfrak{p}_{5}^{3}$ & 3,9 \\
\hline 12439 & -223 & 3 & 27 & $\mathrm{Cl} \mathfrak{p}_{31}, \mathrm{Cl} \mathfrak{p}_{31}^{\prime}$ & $\mathfrak{p}_{31}^{9} \sim 1, \mathfrak{p}_{31}^{\prime 3} \sim \mathfrak{p}_{31}^{3}$ & 3,9 \\
\hline 12439 & 209 & 45 & 27 & $\mathrm{Cl} p_{3}, \mathrm{Cl} p_{3}^{\prime}$ & $\mathfrak{p}_{3}^{9} \sim 1, \mathfrak{p}_{3}^{\prime 3} \sim \mathfrak{p}_{3}^{3}$ & 3,9 \\
\hline 14527 & 164 & 102 & 147 & $\mathrm{Cl} \mathrm{p} p_{2}$ & $p_{2}^{147} \sim 1$ & 3,49 \\
\hline 15561 & -246 & 24 & 243 & $\mathrm{Cl} p_{2}, \mathrm{Cl} \mathfrak{p}_{2}^{\prime}, \mathrm{Cl}_{3}, \mathrm{Cl}_{7}$ & $\mathfrak{p}_{2}^{9} \sim \mathfrak{p}_{3}^{3} \sim \mathfrak{p}_{7}^{3} \sim 1, \mathfrak{p}_{2}^{\prime 3} \sim \mathfrak{p}_{2}^{3}$ & $3,3,3.9$ \\
\hline 15823 & 245 & 33 & 16 & $\mathrm{Clp}_{7}, \mathrm{Cl}_{p_{7}^{\prime}}^{\prime}$ & $\mathfrak{p}_{7}^{4} \sim \mathfrak{p}_{7}^{4} \sim 1$ & 4,4 \\
\hline 15889 & -238 & 48 & 16 & $\mathrm{Cl} \mathrm{p}_{2}, \mathrm{Cl} \mathrm{p}_{2}^{\prime}$ & $\mathfrak{p}_{2}^{4} \sim \mathfrak{p}_{2}^{\prime 4} \sim 1$ & 4,4 \\
\hline
\end{tabular}

(before the reduction) are excessively large in some cases. In the largest case we have encountered, i.e., $(f, a, b)=(15679,-196,90)$, the coordinates of these units with respect to the integral basis $\left\{1, \theta, \theta^{\prime}\right\}$ have about 1400 digits while $\operatorname{Tr}\left(\tau^{-1}\right)$ has 110 digits. Therefore, an efficient method is needed to deal with the large cases if one wants to pursue the computations further along these lines. There are 921 prime conductors less than 16000 and it seems that in most of the very large cases the conductor is one of them.

Much work has been done on unit signatures (see, e.g., [4] and [5]). There are 45 cyclic cubic fields with $f<16000$ such that $\tau$ is totally positive, i.e., $\operatorname{Tr}(\tau)>0$, $\operatorname{Tr}\left(\tau^{-1}\right)>0$. These fields and their class numbers $h$ are listed in Table 1 .

Contrary to the general totally real cubic case [1], the Sylow $p$-subgroups of the class group $G$ are elementary abelian for most fields $K$. This peculiarity of cyclic fields has been elucidated in [10]. In Table 2 we collect all the fields $K$ with $f<16000$ such that the ideal class group $G$ of $K$ has a Sylow $p$-subgroup which is not elementary abelian. For $f=7351$ and 8563 the structure of $G$ has been determined by Smadja [11], and for $f=4711,5383,7657$ the result follows from [3]. By $\mathfrak{p}_{p}$ we denote a prime ideal with norm $\mathrm{N}\left(\mathfrak{p}_{p}\right)=p, \mathrm{Cl} \mathfrak{p}_{p}$ is the ideal class containing it, and $\mathfrak{p}_{p}^{\prime}$ is a conjugate ideal. The symbol $\sim$ indicates equivalence of ideals.

Table 3 gives statistics referring to the class numbers $h$. The numbers at the top of each column are the bounds on the conductor. 


\section{TABLE 3}

\begin{tabular}{|r|r|r|r|r|r|}
\hline$h$ & 1 & 4001 & 8001 & 12001 & \\
\hline 1 & 230 & 186 & 171 & 170 & 757 \\
3 & 249 & 236 & 238 & 227 & 950 \\
4 & 24 & 23 & 23 & 27 & 97 \\
7 & 10 & 4 & 7 & 2 & 23 \\
9 & 64 & 91 & 108 & 101 & 364 \\
12 & 27 & 35 & 32 & 29 & 123 \\
13 & 2 & 1 & 2 & 1 & 6 \\
16 & 1 & 3 & 0 & 2 & 6 \\
19 & 5 & 1 & 1 & 1 & 8 \\
21 & 8 & 14 & 11 & 9 & 42 \\
25 & 0 & 1 & 0 & 1 & 2 \\
27 & 2 & 11 & 11 & 18 & 42 \\
28 & 0 & 3 & 2 & 0 & 5 \\
31 & 0 & 1 & 3 & 0 & 4 \\
36 & 5 & 11 & 7 & 18 & 41 \\
37 & 0 & 0 & 1 & 1 & 2 \\
39 & 1 & 2 & 4 & 10 & 17 \\
43 & 0 & 0 & 1 & 1 & 2 \\
48 & 1 & 2 & 1 & 0 & 4 \\
49 & 0 & 1 & 2 & 0 & 3 \\
57 & 0 & 3 & 0 & 2 & 5 \\
61 & 0 & 0 & 0 & 2 & 2 \\
63 & 1 & 3 & 4 & 3 & 11 \\
64 & 0 & 0 & 1 & 0 & 1 \\
73 & 0 & 0 & 1 & 0 & 1 \\
75 & 0 & 2 & 0 & 0 & 2 \\
81 & 0 & 1 & 0 & 0 & 1 \\
84 & 0 & 1 & 1 & 0 & 2 \\
93 & 0 & 0 & 0 & 1 & 1 \\
109 & 0 & 0 & 1 & 1 & 2 \\
111 & 0 & 0 & 0 & 1 & 1 \\
117 & 0 & 0 & 2 & 1 & 3 \\
127 & 0 & 0 & 0 & 1 & 1 \\
147 & 0 & 0 & 0 & 1 & 1 \\
171 & 0 & 1 & 0 & 0 & 1 \\
228 & 0 & 1 & 1 & 0 & 2 \\
243 & 0 & 0 & 0 & 1 & 1 \\
\hline$\Sigma$ & 630 & 638 & 636 & 632 & 2536 \\
\hline
\end{tabular}

Let $t$ denote the number of ramified primes. It is well-known that the number of ambiguous classes is $3^{t-1}$. For $h=27$, it is not hard to see that the structure of $G$ (even as a $\mathrm{Gal}(K / \mathbf{Q})$-module) is determined by $t$. For $t=2$ we have the 10 cases with $G \cong(\mathbf{Z} / 3 \mathbf{Z}) \oplus(\mathbf{Z} / \mathbf{9 Z})$ listed above. For $t=3$ and 4 there are, respectively, 25 and 7 fields with $G \cong(\mathbf{Z} / 3 \mathbf{Z})^{3}$. In the latter case the action of $\operatorname{Gal}(K / \mathbf{Q})$ on $G$ is trivial, all fields having conductor $f=15561$.

The elementary abelian class group of order 16 occurs for $(f, a, b)=(7687,-169$, 27) and is generated by $\mathrm{Cl} \mathfrak{p}_{3}, \mathrm{Cl} \mathfrak{p}_{3}^{\prime}, \mathrm{Cl} \mathfrak{p}_{13}, \mathrm{Cl} \mathfrak{p}_{13}^{\prime}$.

For $(f, a, b)=(10267,-1,117)$ we have $G \cong(\mathbf{Z} / 7 \mathbf{Z})^{2}$, as has been first shown in [11]. 
It is obvious that a 5-class group of order 25 must be noncyclic. This occurs for $(f, a, b)=(6901,-154,36),(7441,-46,96)$ when $h=75$, and for $(f, a, b)=(7753$, $-58,96),(15937,-190,96)$ when $h=25$. All these groups are generated by $\mathrm{Cl} \mathfrak{p}_{2}$, $\mathrm{Cl} \mathfrak{p}_{2}^{\prime}$ with relations $\mathfrak{p}_{2}^{h / 5} \sim 1, \mathfrak{p}_{2}^{\prime 5} \sim \mathfrak{p}_{2}^{5}$.

All of the computations were done on the DEC-20 computer at the University of Turku, Finland, and we would like to express our gratitude to the members of the staff of the Computer Center for their cooperation. The work has been supported financially by the Academy of Finland.

Department of Mathematics

University of Turku

SF-20500 Turku 50

Finland

1. Veikko Ennola \& Reino Turunen, "On totally real cubic fields," Math. Comp., v. 44, 1985, pp. 495-518.

2. H. J. Godwin, "The calculation of large units in cyclic cubic fields," J. Reine Angew. Math., v. 338 , 1983, pp. 216-220.

3. GeORGES Gras, "Sur les l-classes d'ideaux dans les extensions cycliques relatives de degré premier $l$," I, II, Ann. Inst. Fourier (Grenoble), v. 23, no. 3, 1973, pp. 1-48; ibid., v. 23, no. 4, 1973, pp. 1-44. MR 50 \#12967.

4. GeORges Gras \& Marie-Nicole Gras, "Signature des unités cyclotomiques et parité du nombre de classes des extensions cycliques de $\mathbf{Q}$ de degré premier impair," Ann. Inst. Fourier (Grenoble), v. 25, no. 1, 1975, pp. 1-22. MR 52 \#13728.

5. Marie-Nicole Gras, "Sur le nombre de classes du sous-corps cubique de $\mathbf{Q}^{(p)}, p \equiv 1$ (3)," Séminaire de Théorie des Nombres, 1971-1972 (Univ. Bordeaux I, Talence), Exp. No. 2 bis, 1972, 9pp. MR 53 \#346.

6. Marie-Nicole Gras, "Méthodes et algorithmes pour le calcul numérique du nombre de classes et des unités des extensions cubiques cycliques de Q," J. Reine Angew. Math., v. 277, 1975, pp. 89-116. MR $52 \# 10675$.

7. M. N. Gras, N. Moser \& J. J. Payan, "Approximation algorithmique du groupe des classes de certains corps cubiques cycliques," Acta Arith., v. 23, 1973, pp. 295-300. MR 48 \# 8437.

8. H. HASSE, "Arithmetische Bestimmung von Grundeinheit und Klassenzahl in zyklischen kubischen und biquadratischen Zahlkörpern," Abh. Deutsch. Akad. Wiss. Berlin, Math.-Naturw. Kl., 1948, No. 2. 1950. MR 11, 503.

9. SiRPA MäKI, The Determination of Units in Real Cyclic Sextic Fields, Lecture Notes in Math., vol. 797, Springer-Verlag, Berlin and New York, 1980. MR 82a: 12004.

10. Daniel Shanks, "The simplest cubic fields," Math. Comp., v. 28, 1974, pp. 1137-1152. MR 50 \#4537.

11. Rene Smadja, "Sur le groupe des classes des corps de nombres," C. R. Acad. Sci. Paris Sér. A-B, v. 276, 1973, pp. A1639-A1641. MR 49 \#10661. 